\title{
Preventing violence in youth
}

A fter a wave of violence in Ontario involving students and schools that resulted in the death of 3 teens in 3 separate incidents, Ontario's Minister of Education, Gerard Kennedy, unveiled components of the provincial government's Safe Schools strategy to prevent violence. They include school safety audits, electronic access devices and an anti-bullying hotline.

The components complement earlier ministry initiatives, such as the expansion of the Roots of Empathy program announced by Kennedy in May 2004. This program, which has propagated throughout Canada, aims to enhance emotional literacy and reduce levels of violence by connecting students in elementary classrooms to a neighbourhood family with a baby. Students interact with the baby over 27 instructor-led sessions, exploring themes of human development, cultivating perspective, emotional connection and social inclusion.

Despite their shared goal, the strategies of the Safe Schools and Roots of Empathy programs differ greatly, which illustrates the complexity of violence prevention among the young. It also, perhaps, reveals a lack of evidence-based consensus on the best approach to this important problem.

Violent conduct comprises a wide range of behaviours, from bullying and verbal abuse, through fighting, to rape and homicide. In Canada in 1997, there were 58 homicidal deaths among young men aged 15-24 years, making homicide the fourth leading cause of death (after unintentional injuries, suicide and cancer) in this age group. More recent concerns have been triggered by escalating rates of early childhood aggression, violence perpetrated by female youth and suicide attributed to distress from bullying. ${ }^{1}$

Determinants of violence are multifaceted, complex and even conflicting. They include individual attributes (self-esteem that is either low or very high; lack of learned skills in conflict resolution; scant or even excessive financial resources), familial contexts (poor parenting skills, modelling of bad behaviour, economic stresses and predisposing cultural norms) and social influences (peer norms for dominance and display; low levels of social engagement or involvement in gangs; weak school curriculum; media messages displaying or promoting materialism, aggression or egocentrism; a low level of community safety). Not surprisingly, this complexity has inspired a range of approaches (e.g., ecological, interactionist and developmental) to explain violent behaviour, and various levels of programs to provide interventions (targeted individual, family and social system-focused programs),

not many of which are empirically or evidence-based.

Authors of a recent comprehensive review ${ }^{1}$ of empirically evaluated programs in the United States to prevent youth violence identified 6 critical and recurring issues that appeared to impose obstacles to the success of the programs and that need to be considered when designing such programs. The first pertains to program targeting (i.e., universal v. selected individuals or groups at risk) and the ability of prevention programs to adequately target specific risk and protective factors, especially with time-limited resources and in the context of potential for stigmatization. The second pertains to the extent to which the program is based on sound, empirically derived theory rather than on a "feel-good" reaction to a particular problem. The third and fourth dealt with the extent
Box 1: Key characteristics of programs that are effective against youth violence

Effective programs:

- are comprehensive and multifaceted

- begin in a primary grade

- are developmentally tailored

- include content that promotes personal and social competencies

- make use of interactive techniques to facilitate skills development

- include culturally sensitive material

- ensure "intervention fidelity" through staff development and teacher training

- apply positive (nonaversive) control in the classroom

- foster norms against violence in all school activities
Comment

Family, peers, media and the community should all be considered and included. Program principles should be reinforced across all grade levels.

A risk factor at one age may be protective at another; peer acceptance is a prime example.

Examples include group work, discussions and role-playing.

Material should match the characteristics of the target population(s).

All staff, especially teachers, need to learn how to use and model interactive techniques and conflict management strategies.

This feature is essential. Changing the physical environment, improving maintenance and rethinking schedules can help.

Policies to include are those that prohibit name-calling, graffiti, vandalism, gang colours and symbols, weapons and oversized clothing that can hide weapons. 
Practice

to which the programs were designed with due regard to the culture and the developmental stage (age range) of the target populations. The remaining issues had to do with intervention fidelity (the extent to which the operation and delivery of the program remained faithful to its design) and whether and how the outcome and impact of the programs were assessed.

To identify approaches to school-based violence prevention that are most promising and those that tend to be less effective, in 1997 Dusenbury and colleagues ${ }^{2}$ reviewed the literature and interviewed 15 experts. The result was a list of 9 elements common to effectual violence prevention programs (Box 1) and a shorter list of components that may not work (Box 2).

To fill some of the empirical gaps, the National Center for Injury Prevention and Control, a component of the US Centers for Disease Control and Prevention, has provided funding for

Box 2: Characteristics of programs against youth violence that may not work

- Use of scare tactics that include pictures or videos of violent scenes

- Addition (of the violence prevention program) to a school system that is already overwhelmed

- Segregation of aggressive or antisocial students into a separate group for any purpose

- Instruction that is too brief and lacking the support of a positive school climate

- An exclusive focus on enhancement of self-esteem

- Provision of information and nothing else

an evaluation, in 37 schools over 5 years, of the GREAT (Guiding Responsibility and Expectations for Adolescents for Today and Tomorrow) Schools and Families Program for violence prevention. " Two "universal" interventions are planned for grade 6 students and teachers, along with a targeted intervention for families of students at risk. Details of the program and its evaluation are outlined in a special supplement of the American Fournal of Preventive Medicine. ${ }^{3}$

Erica Weir

CMAf

\section{References}

1. Kerns SE, Prinz RJ. Critical issues in the prevention of violence-related behavior in youth [review]. Clin Child Fam Psychol Rev 2002; 5:133-60.

2. Dusenbury L, Falco M, Lake A, Branningan R, Bosworth K. Nine critical elements of promising violence prevention programs [review]. $7 \mathrm{Sch}$ Health 1997;67:409-14.

3. Multisite Violence Prevention Project. The Multisite Violence Prevention Project: background and overview [review]. Am 7 Prev Med 2004;26 (1 Suppl):3-11

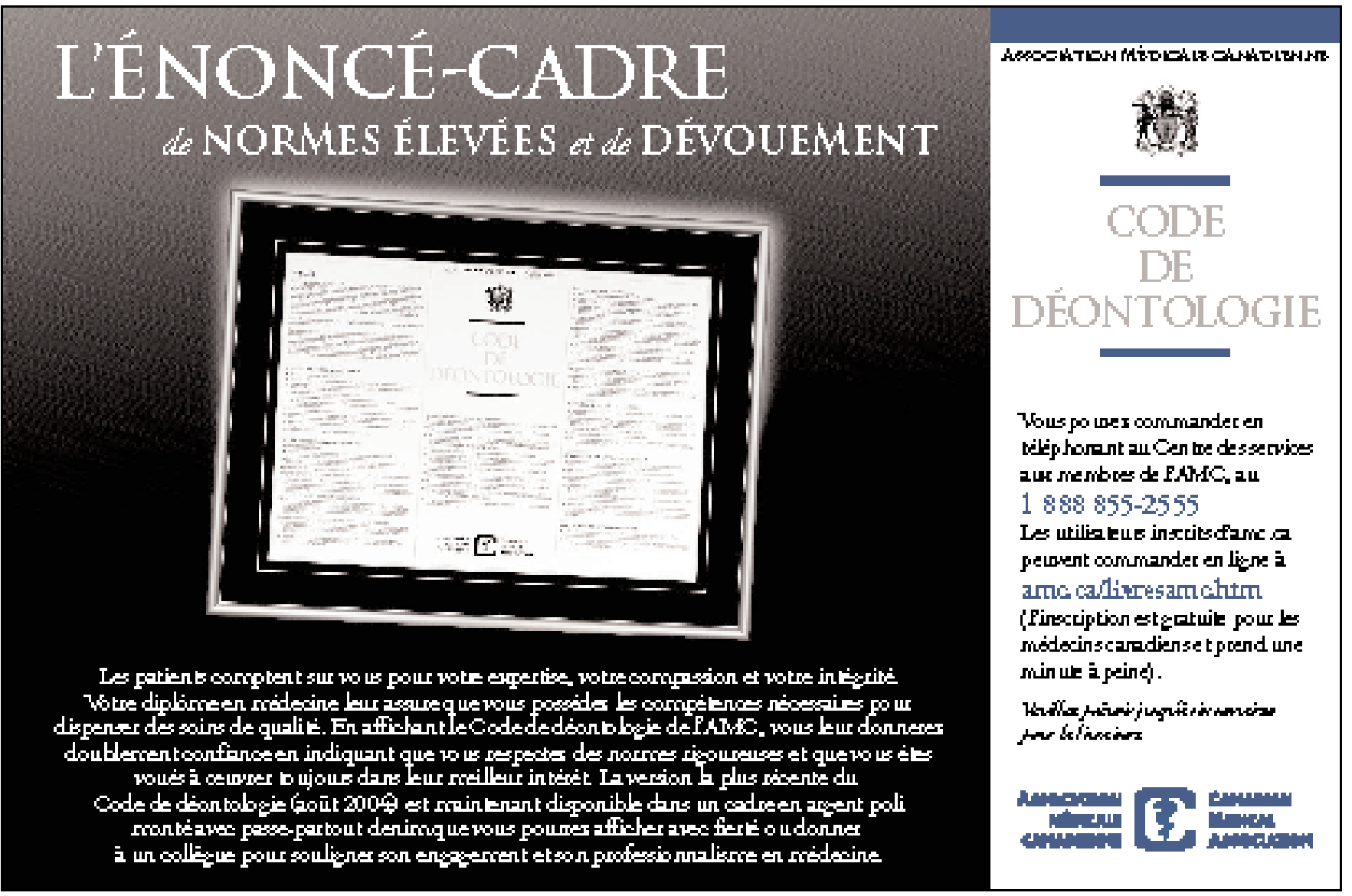

